\title{
Universal (Meta-)Logical Reasoning: Recent Successes
}

\author{
Christoph Benzmüller ${ }^{\mathrm{a}, \mathrm{b}}$ \\ ${ }^{a}$ Freie Universität Berlin, FB Mathematik und Informatik, D-14195 Berlin, Germany \\ ${ }^{b}$ Université du Luxembourg, FSTC, L-4365 Esch-sur-Alzette, Luxembourg
}

\begin{abstract}
Classical higher-order logic, when utilized as a meta-logic in which various other (classical and non-classical) logics can be shallowly embedded, is suitable as a foundation for the development of a universal logical reasoning engine. Such an engine may be employed, as already envisioned by Leibniz, to support the rigorous formalisation and deep logical analysis of rational arguments on the computer. A respective universal logical reasoning framework is described in this article and a range of successful first applications in philosophy, artificial intelligence and mathematics are surveyed.
\end{abstract}

Keywords: computational metaphysics, classical higher-order logic, non-classical logics, automated reasoning

\section{Introduction}

The quest for a universal reasoning framework is very prominently represented in the works of Leibniz. He envisioned a scientia generalis founded on a characteristica universalis, that is, a universal formal language in which all knowledge about the world and the sciences can be encoded. A quick study of the survey literature on logical formalisms suggests that quite the opposite to Leibniz's dream has become reality. Instead of a characteristica universalis, we are today facing a very rich and heterogenous zoo of different logical systems, and instead of converging towards a single superior logic, this logic zoo is further expanding, eventually even at accelerated pace. As a consequence, the unified vision of Leibniz seems farther away than ever before. However, there are also some promising initiatives to counteract these diverging developments, and related works on unifying approaches to logic include categorial logic [1, 2, algebraic logic [3] and coalgebraic logic [4, 5]. While some practical work has been reported utilizing the algebraic logic approach [6, 7, these approaches typically have had a strong emphasis on theory only.

\footnotetext{
This research was funded by the German National Research Foundation (DFG) under Heisenberg grant BE 2501/9 (Studies in Computational Metaphysics) and by Volkswagen Stiftung under grant CRAP (Consistent Rational Argumentation in Politics).

$U R L:$ http://christoph-benzmueller.de (Christoph Benzmüller)
} 
The solution presented here draws on another alternative at universal logical reasoning: the shallow semantical embeddings (SSE) approach. This approach has a very pragmatic motivation, foremost reuse of tools, simplicity and elegance. It utilises classical higher-order logic (HOL) [8, 9] as a unifying metalogic in which the syntax and semantics of varying other logics can be explicitly modeled and flexibly combined. Off-the-shelf interactive theorem provers (ITPs) and automated theorem provers (ATPs) for HOL [10] can then be employed to reason about and within the shallowly embedded logics.

This survey article summarises and reflects upon the main results of the research project Studies in Computational Metaphysics (CompMeta), conducted from 2012 to 2017 at Freie Universität Berlin and Stanford University. In this project the SSE approach has been further developed and empirical studies have been conducted in various disciplines, including philosophy, mathematics and artificial intelligence. In philosophy/metaphysics, for example, an initial focus has been on computer-supported assessments of modern variants of the ontological argument for the existence of God, where the SSE approach has been utilised in particular for automating variants of higher-order (multi-)modal logics [11. It is these sort of challenge applications of expressive logical reasoning that the SSE approach is primarily addressing.

A most relevant aspect from the perspective of computer programming is that the SSE approach in an elegant and theoretically well-founded manner strives for maximal reuse of already existing theorem proving technology with minimal coding effort. The prototype systems that have been implemented in the course of CompMeta performed surprisingly well in all the conducted case studies, which provides good evidence for the practical relevance of the approach. Note that the performance of these implemented systems will (to some extent) naturally advance in the future without much effort simply because the stateof-the-art ATPs and satisfiability modulo theories (SMT) solvers it integrates will further improve in regular cycles.

The article is structured as follows. Section 2 outlines the SSE approach and discusses its application with a challenge puzzle in epistemic reasoning: the wise men puzzle. The presented solution puts a particular emphasis on the adequate modeling of common knowledge. Section 3 presents the motivation and objectives of the CompMeta project, in which the SSE approach has been further explored and empirically assessed. The main results of CompMeta are subsequently summarised and discussed in Sect. 4. Section 5 concludes the article.

\section{The Shallow Semantical Embeddings Approach}

HOL has its roots in the logic of Frege's Begriffsschrift [12. However, the version of HOL as addressed here is a (simply) typed logic of functions, which has been proposed by Church [8]. It provides lambda-notation, as an elegant and useful means to denote unnamed functions, predicates and sets (by their characteristic functions). Types in HOL eliminate paradoxes and inconsistencies. Russel's paradox (the set of sets which do not contain themselves), for 
example, which can be formalized in Frege's logic, cannot be represented in HOL due to type constraints. For more details and further references on HOL and its automation we refer to the literature [9, 10. Very relevant for the work presented here is that the theory of HOL is well understood [13, 14] and that offthe-shelf ATPs and ITPs for HOL exists, which can easily be reused. Respective reasoning systems that are particularly relevant for the SSE approach include the proof assistants Isabelle/HOL [15] and Coq [16], the ATPs LEO-II [17, Leo-III [18] and Satallax [19], and the model finder Nitpick [20. In the running example discussed below the Isabelle/HOL proof assistant is used. There are two main reasons for this choice: (i) The powerful graphical interface of Isabelle/HOL enables a particularly intuitive interaction with the SSE approach in which the logic embeddings can be very elegantly displayed and edited, and (ii) this system, via its Sledgehammer tool [21, integrates powerful first-order (FO) ATPs and SMT solvers, including E [22, CVC4 [23], Z3 [24, SPASS [25] and Vampire [26, and it also connects with the HOL ATPs Leo-II and Satallax. This combination makes it a most suitable environment for conducting mixed interactive and automated experiments with the SSE approach.

In the remainder of this section the SSE approach will be outlined with the help of a prominent puzzle in epistemic reasoning: the wise men puzzle (cf. its discussion in the literature [27, 28]). A particular emphasis and novelty in the formalisation below is the adequate modeling of the common knowledge of a set of agents, which is defined as the transitive closure of the agents mutual knowledge. While the adequate encoding of the notion of transitive closure poses a challenge for inexpressive knowledge representation frameworks, we here utilise and showcase a particularly short and elegant solution in HOL (a single line of code).

\subsection{Outline of the SSE Approach}

Let L be an object logic of interest, for example, higher-order (HO) modal logic, which amongst others has prominent applications in metaphysics. Since our running example below requires the combination of modalities, we will in fact work with a HO multi-modal logic (HOMML).

The overall idea of the SSE approach is to provide a lean and elegant equational theory which interprets the syntactical constituents of logic L (in our case HOMML) as lambda-terms of the meta-logic HOL.

An encoding of HOMML in HOL is presented in Fig.1. Unlike the traditional translation approach 29, the connection between HOMML and HOL, i.e. the equational theory defining the translation is itself formalised in HOL. Moreover, in contrast to a deep logical embedding, where the syntax and the semantics of logic L would be formalised in full detail (using structural induction and recursion), only the crucial differences in the semantics of both, L and HOL, are directly addressed in the equational theory, while the commonalities are shared between both logics. HOMML and HOL, for example, share the domain of individuals. A crucial difference, however, lies in the possible world semantics on the side of HOMML. Hence, the equational theory defining HOMML in HOL provides an explicit modeling of this particular aspect of modal semantics. The 


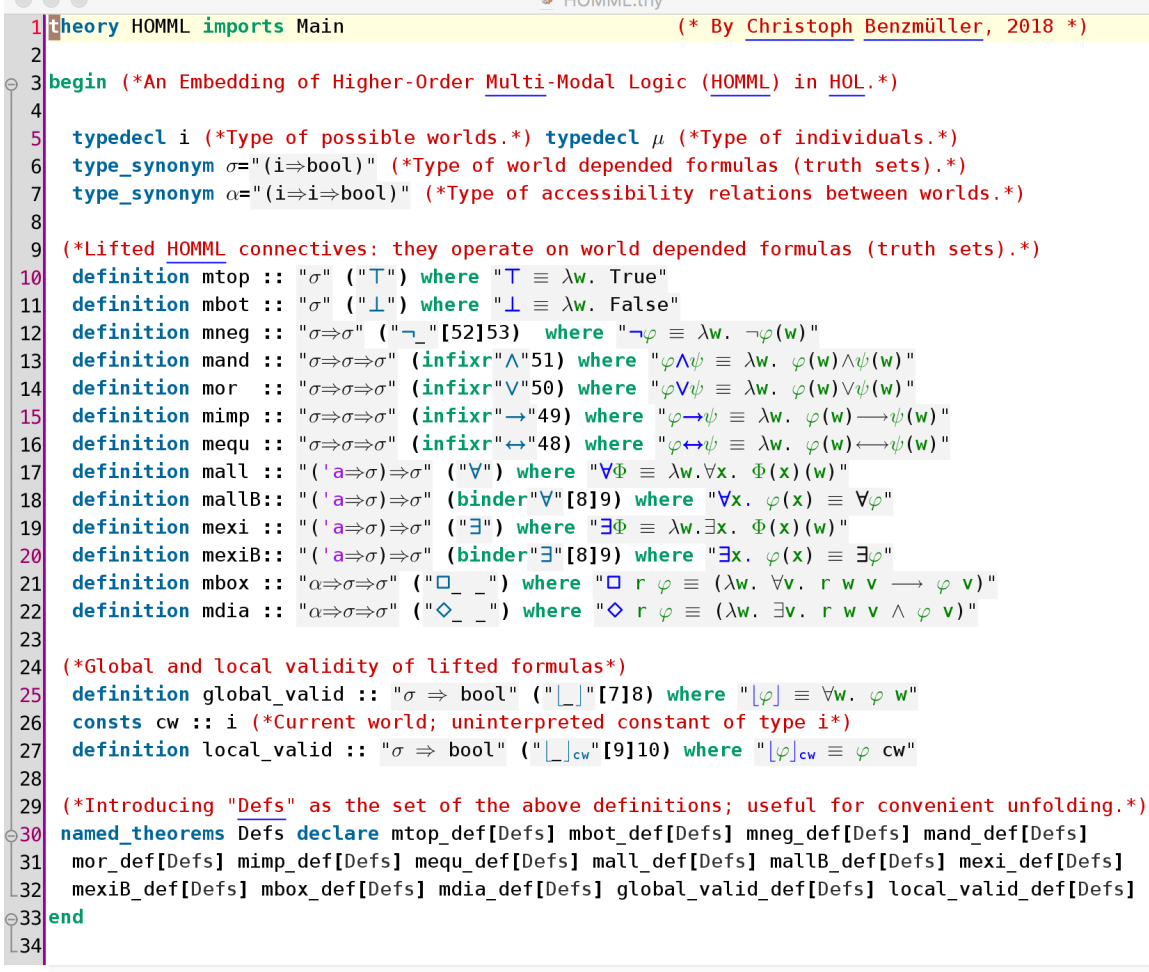

Figure 1: Shallow semantical embedding of HOMML in HOL.

central idea of this theory is to associate Boolean valued formulas $\varphi_{o}$ of HOMML with world-predicates (truth-sets characterised as lambda-abstractions) $\varphi_{i \rightarrow o}$ in HOL, where $i$ stands for a reserved type for worlds (lines 5-7 in Fig. 1). The predicate type $i \rightarrow o$ is abbreviated as $\sigma$ and $\alpha$ stands for the type $i \rightarrow i \rightarrow o$ of accessibility relations.

To establish our mapping it essentially suffices to equate the classical logical connectives of HOMML with corresponding world-lifted predicates and relations in HOL. For example, in line 13 in Fig. 1 the HOMML connective $\wedge$ is identified with the lambda-term $\lambda \varphi \cdot \lambda \psi \cdot \lambda w \cdot \varphi w \wedge \psi w$, such that $\varphi \wedge \psi$ is mapped to the truth set $\{x \mid \varphi w \wedge \psi w\}$, which is denoted in HOL by the lambda-term $\lambda w . \varphi w \wedge \psi w$. The indexed modal operators $\square r$ of HOMML are identified with lambda-terms $\lambda \varphi . \lambda w . \forall v . r w v \rightarrow \varphi v$, where argument symbol $r$ denotes an accessibility relation between possible worlds. We may alternatively say that a parameterised, generic $\square$-operator is introduced here as $\lambda r \cdot \lambda \varphi \cdot \lambda w \cdot \forall v \cdot r w v \rightarrow \varphi v$, such that $\square r \varphi$ is mapped to the truth set $\lambda w . \forall v . r w v \rightarrow \varphi v$ (line 21 in Fig. 1). This generic $\square$-operator can then be instantiated for concrete accessibility relations $r$ as required.

The mapping of constant symbols and variables from HOMML to HOL is 
trivial, since only a type-lifting is required. Most importantly, the mapping of HOMML to HOL can be defined by a set of non-recursive equations (in fact, abbreviations), in which the dependency of HOMML formulas on possible worlds is made explicit, while other aspects and parameters of its semantic interpretation, such as the underlying semantic domains, remain shared between HOMML and HOL.

Another interesting and important aspect is that the SSE approach scales well for $\mathrm{FO}$ and $\mathrm{HO}$ quantifiers. Analogous to the encoding of the propositional HOMML connectives, they can be introduced as simple abbreviations of lambda-terms in HOL as well. The introduction of new binder mechanisms is not required, since the already existing lambda-binder(s) in meta-logic HOL can be elegantly reused. This is shown in line 17 in Fig. 11 where the HOL universal quantifier $\Pi_{(\mu \rightarrow o) \rightarrow o}$ is type-lifted to become a HOMML universal quantifier $\boldsymbol{\Pi}_{(\mu \rightarrow(i \rightarrow o)) \rightarrow(i \rightarrow o)}$. In line 18 convenient binder notation is then defined for the lifted HOMML quantifier, so that we may write $\forall x . \phi x$ instead of the less intuitive variant $\Pi(\lambda x . \phi x)$. Type polymorphism is employed in lines $17-18$ to avoid the otherwise required enumeration of such quantifier-defining equations for different argument types. The existential quantifier for HOMML is introduced analogously in lines 19-20.

The final step is to provide a notion of validity for the type-lifted HOMML formulas in HOL (see line 25 in Fig. 1): A type-lifted formula $\varphi$, is valid, denoted here as $\lfloor\varphi\rfloor$, if and only if the application of $\varphi$ to $w$ holds for all worlds $w$. In addition, a notion of local validity, denoted here as $\lfloor\varphi\rfloor_{c w}$, can be introduced: $\lfloor\varphi\rfloor_{c w}$ is true if and only if $\varphi c w$ holds, where $c w$ is an uninterpreted constant symbol representing the current world.

The presented equations thus characterise a fragment of HOL which, modulo the above sketched type-lifting, corresponds to HOMML. The faithfulness of this correspondence, that is, its soundness and completeness with respect to Henkin semantics, can be established with pen and paper methods [1].1]

\subsection{Operations on (Accessibility) Relations, including Transitive Closure}

Figure 2 presents some useful operations on (accessibility) relations. They can e.g. be used to elegantly postulate an accessibility relation $r$ to be reflexive, transitive and euclidean, which are the semantic properties typically associated with modal knowledge operators $\square r$ (see also line 6 in Fig. 3). The expressivity of HOL is particularly exploited in the single-line definition of the transitive closure operation $t c$ in line 14 in Fig. 2, which expresses that two objects (worlds) $x$ and $y$ are related in the transitive closure $(t c R)$ of a relation $R$ if and only if they are related in all transitive super-relations $Q$ of $R$. Utilising this definition, ATPs integrated with Isabelle/HOL via Sledgehammer can be employed to prove some useful lemmata, including the transitivity of the transitive closure

\footnotetext{
${ }^{1}$ Further work will investigate whether such faithfulness proofs can eventually be formalised as well in the approach presented here or whether e.g. a deep embedding is required in this case.
} 


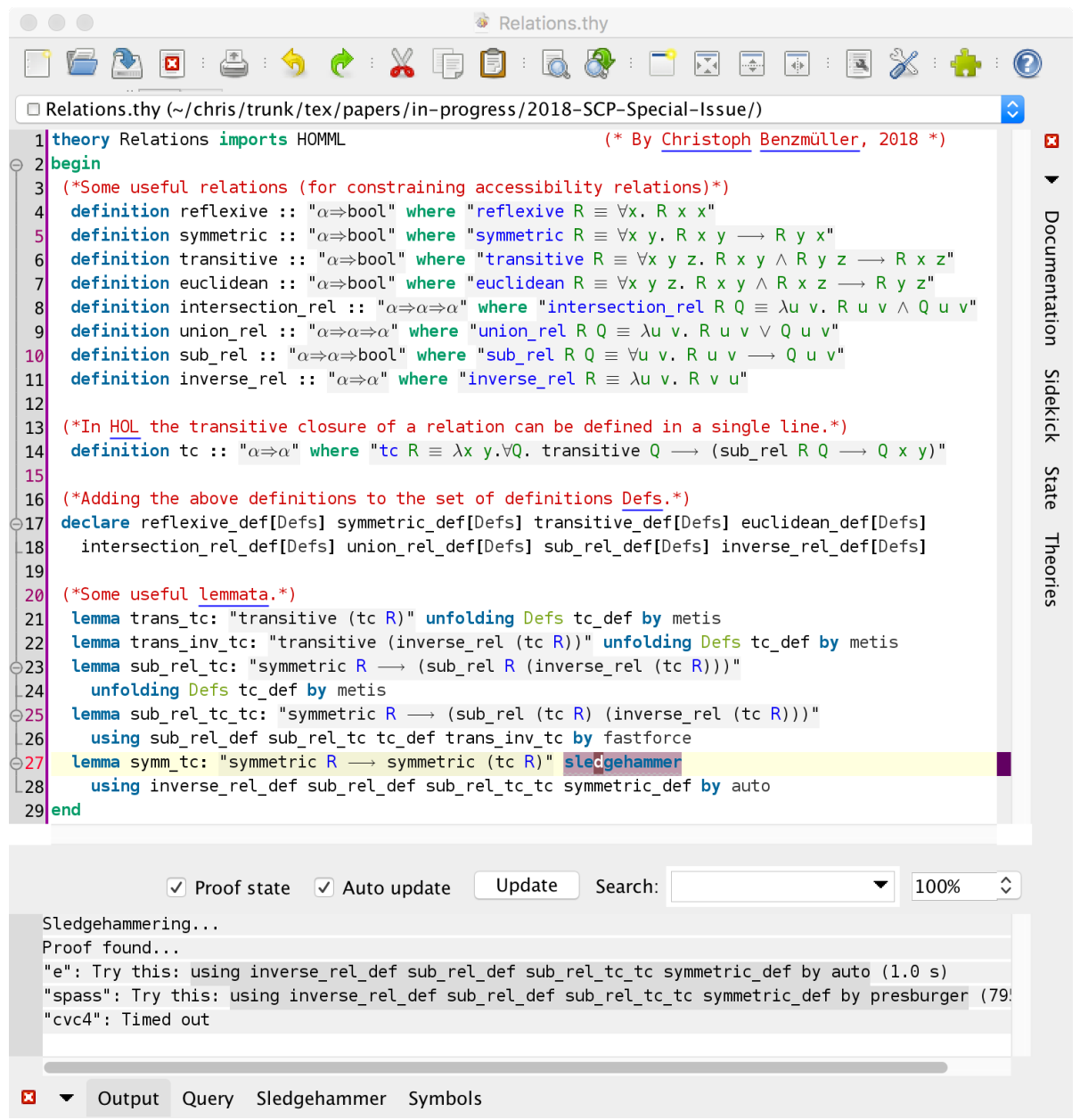

Figure 2: Operations on (Accessibility) Relations, including Transitive Closure. 
of any relation $R$ (line 21) and the symmetry of the transitive closure of any symmetric relation $R$ (lines 27-28). In the lower window of the GUI in Fig. 2 we e.g. see that the FO ATPs E and SPASS quickly prove the latter lemma, while the SMT solver CVC4 timesout (the SMT solver Z3 is still running here). Sledgehammer determines the exact dependencies for the proven conjecture and it identifies a trusted proof tactic in Isabelle/HOL (here auto), which is capable of reproving the lemma when the determined dependencies are preselected. The reconstructed proof utilising the tactic auto is recognised by Isabelle's inference kernel. The lemma is then accepted as such by the system.

\subsection{Example Application: Wise Men Puzzle}

The wise men puzzle, a famous logic riddle whose formalisation has been studied in some detail e.g. by Baldoni [27, is as follows: Once upon a time, a king wanted to find the wisest out of his three wisest men. He arranged them in a circle and told them that he would put a white or a black spot on their foreheads and that one of the three spots would certainly be white. The three wise men could see and hear each other but, of course, they could not see their faces reflected anywhere. The king, then, asked to each of them to find out the color of his own spot. After a while, the wisest correctly answered that his spot was white.

An encoding of this epistemic puzzle scenario utilising the SSE approach is presented in Fig. 3. As an improvement over related work and also over own previous experiments [30, an adequate modeling of mutual knowledge and common knowledge is provided in Fig. 3 by following the suggestions of Sergot [31. The key idea is to model the knowledge of each wise men, say $a$, with the help of an indexed KT45 (=S5) modal operator $\square a$. We thus introduce three accessibilty relations $a, b$ and $c$ (see line 3 in Fig 3 ) and instantiate the generic $\square$-operator from Fig 1 accordingly to obtain the indexed knowledge operators $\square a$, $\square b$ and $\square c$, one for each wise men (cf. their uses in lines 26,28 and 30). The accessibilty relations $a, b$ and $c$ are constrained in line 6 to obey reflexivity, transitivity and euclideaness. This ensures that $\square a, \square b$ and $\square c$ are KT45 knowledge operators as intended. Following Sergot, the mutual knowledge of the wise men $a, b$ and $c$ is introduced next be defining a relation Eabc as the union of the accessibility relations $a, b$ and $c$ (in line 8). However, the corresponding $\square E a b c$-operator does not yet qualify as an operator for common knowledge, since it may fail to be transitive. Hence, another relation $C a b c$ is introduced as the transitive closure of Eabc. The ATPs integrated with Isabelle/HOL confirm (in lines 12-14) that $C a b c$ is reflexive, transitive and euclidean, which means that $\square C a b c$ is a suitable encoding of the common knowledge of the wise men $a, b$ and $c$.

The formalisation of the epistemic puzzle scenario is continued in lines 1630 in Fig. $\left.3\right|^{2}$ In addition to the already declared constant symbols $a, b$ and

\footnotetext{
${ }^{2}$ This encoding still abstracts from the temporal dynamics of the scenario, and the adequate inclusion of such aspects, for example by adopting and integrating an semantic embedding of dynamic epistemic logic [32, is still ongoing work. The formalisation presented here never-
} 


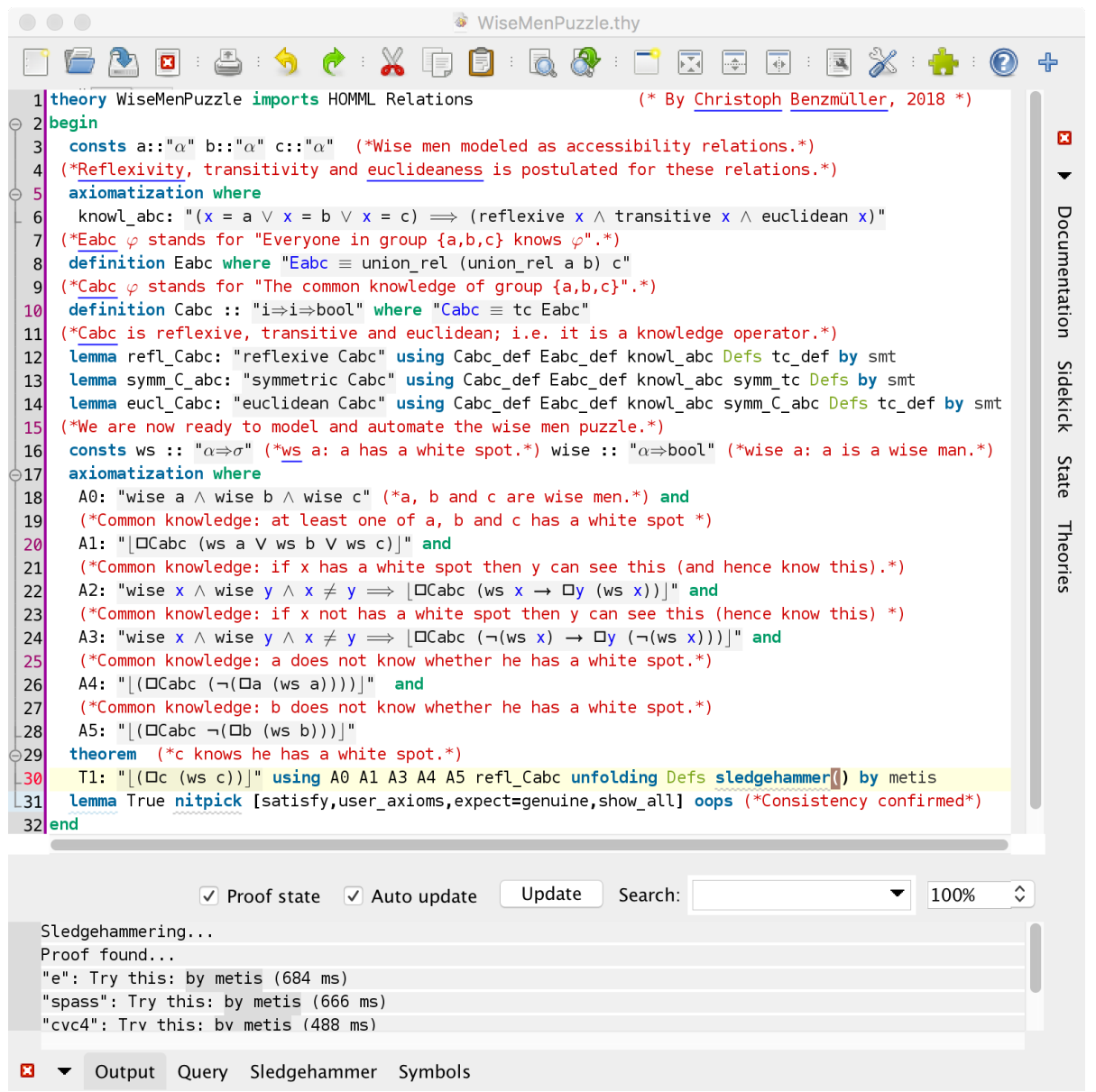

Figure 3: Wise Men Puzzle. 
$c$, which denote the epistemic accessibility relations of the wise men in the scenario, two further uninterpreted constants symbols are introduced in line 16 of Fig. 3. Predicate wise is used to identify and denote the set of wise men in the scenario (see line 18), and the predicate $w s$ expresses whether a wise man has a white spot. Line 20 states that it is common knowledge of the wise men $a, b$ and $c$ that at least one of them has a wite spot. Line 22 (respectively, line 24) then postulates that it is common knowledge that if one wise man has a white spot (respectively, not a white spot), then the other wise men see and thus know this. This information, which is implicit background knowledge that is not explicitly stated in the puzzle itself, is nevertheless relevant for solving it. Note in particular, how the exploitation of the meta-logic HOL here avoids the otherwise required duplication of these axioms for different combinations of $x$ and $y$ in the object logic HOMML. Lines 26 and 28 encode the information that the first two wise men that are asked by the king (they are called $a$ and $b$ here) do not know whether they have a white spot. This again is postulated as common knowledge of the wise men. Then, in line 30, the theorem is formulated that the third wise men $c$ now knows that he has a white spot. This theorem can be proven by the ATPs integrated with Isabelle/HOL via the Sledgehammer tool. The lower part of the GUI window in Fig. 3 shows that the FO ATPs E and SPASS succeed, and so does the SMT solver CVC4 (while Z3 is still running). Moreover, in line 31, a consistency check for the entire formalisation of the puzzle scenario is performed: the model finder Nitpick computes and presents a model (not displayed here) that satisfies the presented axioms and definitions.

Note the elegance and minimal effort with which an integrated interactive and automated theorem proving environment for HOMML has been implemented in Figures 1, 3 on top of an existing theorem proving infrastructure for HOL. In fact, the entire implementation of HOMML and its application to the wise men puzzle did not require more than 94 line of Isabelle/HOL code, including commentary. And still, a good degree of automation is achieved, which significantly benefits from the existing ATPs and SMT solvers already integrated with Isabelle/HOL via the Sledgehammer tool.

After this brief illustration of the SSE approach we now turn attention to the CompMeta project and briefly discuss its original motivation, its objectives, and some relevant preceding work.

\section{Objectives of the CompMeta Project and Preliminary Work}

The CompMeta project has its intellectual roots in the author's work, conducted with colleagues since the mid-nineties, on the theory and practice of HO theorem proving (cf. 14, 33, 34 and the references therein), on HO proof assistants (cf. 35, 36] and the references therein), and on their applications in mathe-

theless already elegantly demonstrates some core advantages of the SSE approach, including the already mentioned appropriate modeling of common knowledge based on the transitive closure of mutual knoweldge. 
matics, artificial intelligence and education (e.g. [37, 38, 39]). These research activities inspired first experiments towards the development of a universal (meta-) logical reasoning framework based on the SSE approach [40, 41, 30, 11]. The core motivation for the CompMeta project has been to further consolidate these initial ideas and to assess the approach in empirical studies. The main objectives thus included

1. to further explore the theoretical foundation of the SSE approach,

2. to exemplarily implement the approach for a range of challenge logics in existing ATPs and ITPs for HOL,

3. to provide evidence for its universal logical reasoning capabilities within exemplary case studies in metaphysics and beyond,

4. and to educate a new generation of students and researchers to master the SSE approach.

Regarding (1) it was planned to study the faithfulness of the embedding of further challenging quantified non-classical logics in HOL. The hypothesis has been that in all cases the faithfulness, i.e. soundness and completeness of the embedding, can be shown when a notion of Henkin semantics is assumed (on the side of both logics). Regarding (2), a close collaboration with the projects Leo-II and Leo-III, running in parallel at Freie Univerität Berlin, was foreseen, in addition to the use of the proof assistants Isabelle/HOL and Coq. With respect to (3), a focus has been on applications in theoretical philosophy, resp. in metaphysics, since there is a particular need for very expressive non-classical logics in this area. For example, hyper-intensional second order modal logic is utilised as the starting point in Zalta's Principia Logico-Metaphysica 42, and similarly expressive logics are studied in prominent recent textbooks by Stalnaker [43] and Williamson [44. Unfortunately, however, there had not been any attempts prior to the CompMeta project to implement and automate such challenging logic formalisms in computer systems. Mainstream knowledge representation formalisms in computer science and artificial intelligence, including e.g. semantic web technologies, typically fail to deliver (not only) in this application context due to their lack of expressivity. The CompMeta project, in contrast, intended to address this gap and to contribute to the pioneering of the new area of computational metaphysics, which has its roots in the work of Zalta and colleagues at Stanford University [45, 46, 47. For the appropriate modeling of foundational philosophical ontologies and for the formal analysis of challenge arguments in philosophy (and beyond) a suitably expressive modeling and reasoning framework was obviously required. With respect to (4), the goal has been to design and offer an interdisciplinary lecture course on computational metaphysics, in which the active use of the CompMeta framework was intended to play a central role in combination with the training of a new generation of students to independently master the approach. 


\section{Results of the CompMeta Project}

The main results and highlights of the CompMeta project are summarised in this section and references are given to the most important publications stemming from the project.

\subsection{Application Study I: Ontological Argument for the Existence of God}

Different modern variants of the ontological argument for the existence of God, one of the still vividly debated masterpiece arguments in metaphysics (see e.g. Sobel's textbook [48, and the references therein), have been rigorously analysed on the computer in the course of CompMeta. These contributions, many of which were achieved in close collaboration with Bruno WoltzenlogelPaleo, received a media repercussion on a global scale $3^{3}$

In the course of the conducted experiments 49, 50, 51, 52, the theorem prover Leo-II detected a previously unknown inconsistency in Kurt Gödel's prominent, HO modal logic variant [53] of the ontological argument, while Dana Scott's amendment [54] of it was verified for logical soundness in the interactive proof assistants Isabelle/HOL [15] and Coq 55, In Fig. 4 the axioms causing the inconsistency in Gödel's manuscript are highlighted (see also the discussion in Sect. 4.6.1). This inconsistency, which was missed by philosophers, is explained in detail in two conference papers [51, 50. Further relevant insights contributed or confirmed by ATPs e.g. include the separation of relevant from irrelevant axioms, the determination of mandatory properties of modalities, and undesired side-implications of the axioms such as the modal collapse ${ }^{5}$

Further variants of Gödel's axioms were proposed by Anderson, Hájek and Bjørdal [57, 58, 59, 60, 61, 62. These variants have meanwhile also been formally analysed, and ATPs have even contributed to the clarification of an unsettled philosophical dispute between Anderson and Hájek 63. In the course of this work, different notions of quantification (actualist and possibilist) have been utilised and combined within the semantical embedding approach 64. Moreover, the modal collapse, whose avoidance has been the key motivation for the contributions of Anderson, Bjørdal and Hájek (and many others), has been further investigated [65].

A significant further contribution has been achieved by David Fuenmayor, a philosophy student recruited from in the computational metaphysics lecture

${ }^{3}$ See e.g. http://www.spiegel.de/international/germany/scientists-use-computer-tomathematically-prove-goedel-god-theorem-a-928668.html

${ }^{4}$ Scott was not aware of the inconsistency in Gödel's variant. Amongst others, he slightly modified Gödel's definition of essence, which causes the inconsistency. Scott did so because it felt natural to him to require that essential properties of an individual should actually be possessed by that individual, and so he added a respective conjunct to the definition; cf. also [50, 51.

${ }^{5}$ The modal collapse 56,48 is a sort of constricted inconsistency at the level of possible world semantics. The assumption that there may actually be more than one possible world is refuted; this follows from Gödel's axioms as the ATPs quickly confirm. In other words, Gödel's axioms, as a side-effect, imply that everything is determined (we may even say: that there is no free will). 

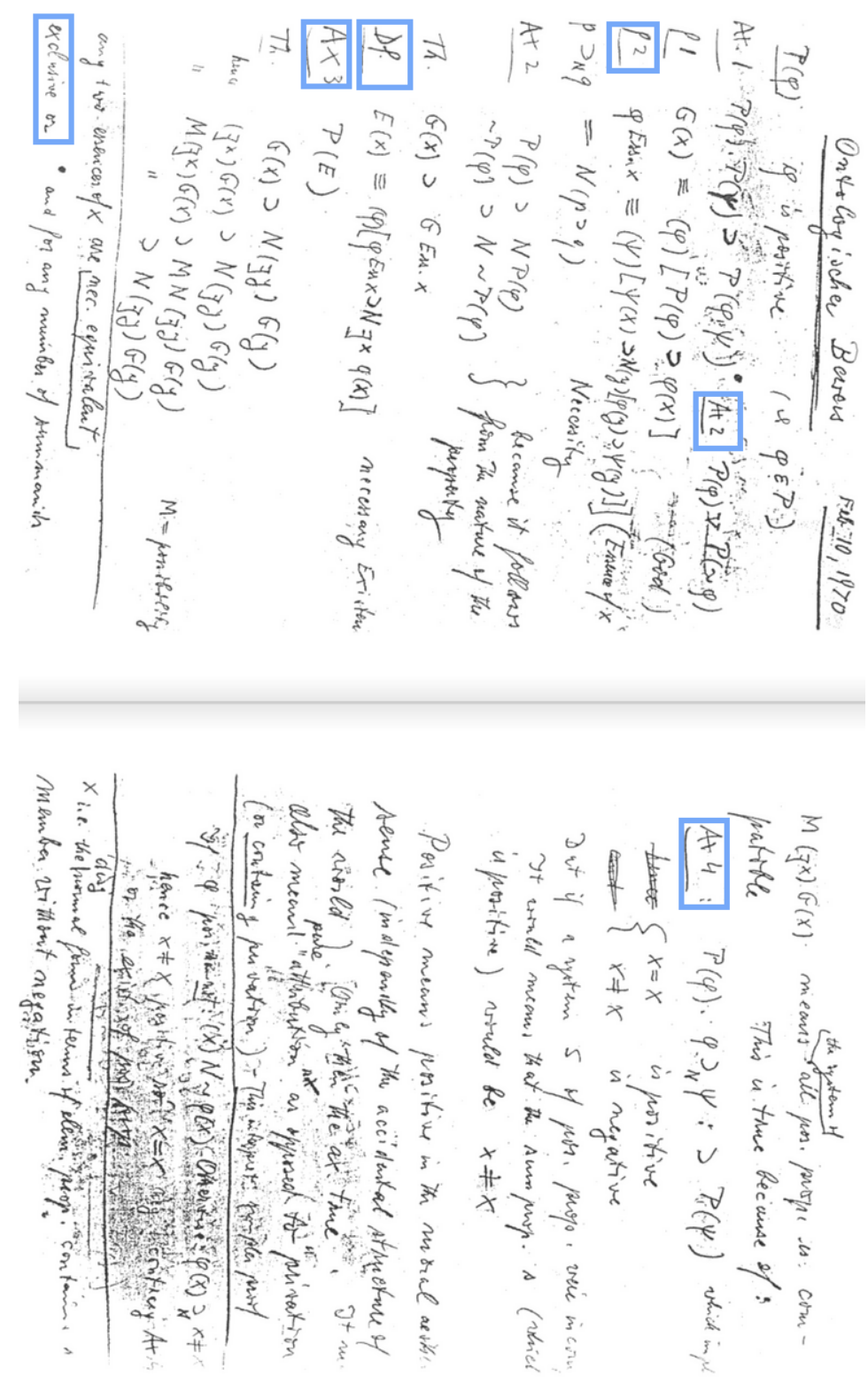

Figure 4: The axioms causing the inconsistency in Gödel's modal logic variant of the ontological argument for the existence of God are highlighted in blue. The inconsistency was detected by the HO ATP Leo-II. (Disclaimer: Unpublished works of Kurt Gödel are Copyright Institute for Advanced Study and are used with permission. All rights reserved by Institute for Advanced Study) 
course at Freie Universität Berlin (see Section 4.5). Fuenmayor, in a student project 66, 67, formalised the most relevant parts of Fitting's 68 textbook Types, Tableaus, and Gödel's God. This book develops another interesting emendation of the ontological argument, which - similar to other recent works - aims at preserving the overall conclusion (necessary existence of God), while at the same time getting rid of the modal collapse. Fitting's means to achieve this is by modifying the foundational logical system. Instead of an extensional HO modal logic he employs a more expressive intensional HO modal logic, which enables a different, and as Fitting explains, more adequate interpretation of e.g. the notion of positive poperties in Gödel's argument.

The so far mentioned studies only address a small portion of the entire relevant literature on the ontological argument. By extending these studies, it can be expected that many further issues in human refereed contributions can be revealed. A follow-up project could thus try to develop an encompassing map that rigorously distinguishes sound from unsound work in this area.

Summary of key insights. Variants of extensional and intensional HO modal logics can easily be implemented in the SSE approach; a very good degree of proof automation can be achieved this way, matching or exceeding the argumentation granularity we typically find in human authored publications on this subject; flexible logic modifications and combinations are supported; the approach is practically highly useful and it combines automated theorem proving with model and countermodel finding (the latter well supports the detection of typos and minor issues during the formalisation process); it has been demonstrated how the approach supports a novel, experimental style of work in metaphysics.

\subsection{Application Study II: Zalta's Principia Logico-Metaphysica}

Formalising and automating masterpiece rational arguments in philosophy with the SSE approach on the computer is not trivial. However, it still leads to comparably small corpora of axioms, lemmata and theorems, and, hence, it does not provide reliable feedback on the scalability of the approach for larger and more ambitious formalisations. For that reason another challenge has been tackled in the CompMeta project: the Principia Logico-Metaphysica (PLM) of Edward Zalta [2], which aims at a foundational logical theory for metaphysics, mathematics and the sciences (PLM thus intends to subsume the Principia Mathematica 69]). Zalta has chosen a hyper-intensional, relational second-order modal logic S5 [70, 71] as the foundational logic for PLM. It has thus been a challenge question for CompMeta, whether this non-trivial foundational logic can still be suitably encoded and automated in the semantical embedding approach. Besides hyper-intensionality, a particular challenge has been to overcome the conceptional gap between the relational core of PLM and the functional core of HOL, and to suitably handle the different strengths of comprehension principles supported in both logics that assert the existence of relations and functions (the use of unrestricted comprehension principles in PLM causes undesirable paradoxes and inconsistencies 72]). And, of course, a main challenge has also been to deal with the comparably large size of PLM 
in relation to the small axiom sets as studied in the context of the ontological argument.

The author's initial attempts, conducted during an extended research stay at Stanford University in 2015/16, to semantically embed PLM's base logic in HOL by following a pure proof theoretic approach were unsuccessful. Later in 2016, in the course of the computational metaphysics lecture course in summer 2016 at Freie Universität Berlin (see below), Zalta in an invited presentation then outlined some ideas towards a set theoretical semantics for PLM, which were suggested to him by Peter Aczel. This set theoretic perspective on PLM subsequently enabled the development of a suitable shallow semantical embedding of PLM in HOL. It was in fact Daniel Kirchner, a mathematics student recruited the lecture course, who took on the challenge within an MSc thesis project at Freie Universität Berlin. Kirchner has meanwhile succeeded in formalising the PLM in Isabelle/HOL by suitably adapting the SSE approach so that it soundly covers the base logic of PLM [73].

Kirchner's work contributes various novel ideas and tools, including the provision of powerful automation means for PLM at different, cross-linked levels of abstraction. For example, he developed a direct, tactic-based theorem prover for PLM in Isabelle/HOL, which, one-to-one, implements the proof theory of PLM as developed by Zalta with pen and paper. This object-level theorem prover for PLM is connected with the HOL meta-level in Kirchner's work via the specifically tailored shallow semantical embedding he developed, and this link establishes an Isabelle/HOL-internal criterion, modulo expansion of the semantical embedding, for the soundness of his novel prover. Further, similar provers are provided by him at well-defined, intermediate expansion levels. Kirchner's architecture thus provides multiple options for proof automation, ranging from the full expansion of the semantical embedding (combined with calls to off-theshelf reasoning tools integrated with Isabelle/HOL via the Sledgehammer tool) to the more intuitive, one-to-one automation of the proof theory of PLM within Kirchner's new tactic-based theorem prover.

An unexpected, but key result of Kirchner's work has been the discovery of a paradox in PLM [74, 73] (in the spirit of Russel's paradox [75] for Frege's logic of the Begriffsschrift [76]): a deeply-rooted and known paradox is reintroduced in PLM, respectively, in the abstract object theory underlying the PLM, when the logic of complex terms is simply adjoined to the frameworks speciallyformulated comprehension principle for relations. Kirchner's result constitutes a new and important paradox, given how much expressive and analytic power is contributed by having the two kinds of complex terms in the system. The results also provide a fresh perspective on the question of whether relational type theory or functional type theory better serves as a foundation for logic and metaphysics 72 .

In close collaboration and supported by further experiments with Isabelle/HOL, possible emendations of PLM are currently being studied by Zalta and Kirchner. The ongoing style of interaction well illustrates a new dynamics in the scientific discovery process in metaphysics: rigorous experimentation with implementations of foundational logical systems may quicken and inspire 
the scientific discovery process in this area and also foster more reliable results.

Summary of key insights. The semantic embedding approach scales for ambitious and large projects in metaphysics such as PLM; the approach is practically applicable and already shows a good degree of automation, which will naturally further improve (with the ATPs it relies upon); with the help of the implemented framework new knowledge has been contributed; moreover, students can be well motivated when using the approach to dive into complex, foundational questions on the edge of current research in metaphysics in short time.

\subsection{Application Study III: Free Logic and Axioms Systems for Category Theory}

Partiality and undefinedness are prominent challenges in various areas of mathematics and computer science. Unfortunately, however, modern proof assistant systems and ATPs based on traditional classical or intuitionistic logics provide rather inadequate support for these challenge concepts. Free logic [77, 78, 79, 80, offers a theoretically appealing solution, but it has been considered as rather unsuited towards practical utilisation.

In collaboration with Dana Scott, a shallow embedding of free logic in HOL has been developed and implemented in the CompMeta project. Just as for the embeddings mentioned above, various state-of-the-art FO and HO ATPs and model finders, which are integrated (modulo suitable logic translations) with Isabelle/HOL via the Sledgehammer tool, can now be utilised to automate reasoning in free logic. As a result we obtain an elegant and powerful implementation of an integrated interactive-automated theorem proving (and model finding) environment for free logic.

To demonstrate the practical relevance of this new system, a series of axioms systems for category theory has been systematically explored [81, 82, 83]. The starting point has been a generalisation of the standard axioms for a monoid to a partial composition operation. The purpose of this work has not been to make or claim any contribution to category theory but rather to show how formalisations involving the kind of logic required, in this case free logic, can be implemented and validated within modern proof assistants such as Isabelle/HOL when utilising the SSE approach.

Subsequently, the relation of the developed axiom systems to alternative proposals from the literature has been studied within the framework. This includes an axiom set proposed by Freyd and Scedrov in their textbook Categories, Allegories [84] for which we have revealed a technical flaw. Either all operations, e.g. morphism composition, are total in their theory or their axiom system is inconsistent. This observation applies when a free logic reading of their axiomatic theory is adopted, where the free variables are assumed to range over all objects, including the "undefined". When adopting an algebraic reading of their axioms system, where free variables range only over all defined objects, then strictness axioms or conditions are missing in their framework. Both readings have been formalised in the SSE approach.

Thus, in interaction with the SSE based implementation of free logic in Isabelle/HOL, a minor (one may say technical) but nevertheless relevant issue 
in a mathematics textbook has been revealed that domain experts had missed before. The repair for this problem is quite straightforward, however. The solution essentially corresponds to a set of axioms proposed by Scott [85] in the 1970s.

In the experiments reported above, the exploration studies have been significantly supported by series of experiments in which automated reasoning tools were called from within the proof assistant Isabelle/HOL via the Sledgehammer tool. Moreover, very useful feedback was obtained at various stages from the model finder Nitpick [20], saving us from making several mistakes.

At the conceptual level this work exemplifies a new style of explorative mathematics which rests on fruitful human-machine interaction with integrated interactive-automated theorem proving technology. The conducted experiments were such that the required reasoning was often too tedious and time-consuming for humans to be carried out repeatedly with highest level of precision. It is here where cycles of formalisation and experimentation efforts in Isabelle/HOL provided significant support. Moreover, the technical inconsistency issue for the axiom system of Freyd and Scedrov was discovered by ATPs, which further emphasises the added value of automated theorem proving in this area.

Summary of key insights. The SSE approach is applicable also to free and inclusive logics, which so far were believed to be too difficult to automate and thus of little practical relevance; quite to the contrary: as our experiments show, the approach is indeed well suited for practical applications, e.g. for the exploration of mathematical theories in domains such as category theory, where partiality and undefinedness play a central role; new knowledge can be discovered this way.

\subsection{Theoretical Study IV: Universal Cut-Elimination}

The development of cut-free calculi for expressive logics, such as quantified non-classical logics, is usually a non-trivial task. However, for a wide range of logics there exists a surprisingly elegant and uniform solution: simply utilise the SSE approach. More precisely, by modeling and studying these logics as semantically embedded fragments of HOL (with Henkin semantics), existing cut-elimination results for HOL (with Henkin semantics) may be reused. In the course of the CompMeta project, this idea has been further explored and exemplarily applied for proving cut-elimination for quantified conditional logics [86].

Conditional logics [87, 88, known also as logics of normality or typicality, have many applications, including counterfactual reasoning, default reasoning, deontic reasoning, metaphysical modeling, action planning and reasoning about knowledge. Moreover, it is well known that they subsume normal modal logics, since the modal box operator can be defined in terms of the more expressive conditional operator. In contrast to the rather straightforward, Kripke-style semantics of normal modal logics, conditional logics come e.g. with a HO selection function semantics, which makes them interesting objects of study. While there is broad literature on propositional conditional logics, comparably few 
authors have addressed FO extensions of conditional logics; those include Delgrande [89, 90, and Friedman et al. 91].

The conditional logics studied in the course of the CompMeta project utilise constant- and/or varying-domain FO quantifiers and they combine these with further quantifiers for propositional variables. Such a rich combination has not been adressed in the literature before. In particular, cut-elimination for these logic(s) was still open (only for propositional conditional logics some related results had been available 92, 93; cf. also the references therein).

While earlier, practical work in the CompMeta project had already shown that automation of quantified conditional logics is indeed feasible by utilising the SSE approach 94, the second half of the project then switched the attention to the theoretical challenge of proving cut-elimination. It was then shown [86, that by utilising the SSE approach for quantified (and non-quantified) conditional logics, the question whether cut-elimination holds for them can in fact be reduced to proving the faithfulness of their semantical embedding in HOL. The latter task, however, constitutes a much simpler problem than proving cut-elimination directly.

The exploited reduction principle is similarly applicable to other object logics in the SSE approach, including many logics for which cut-elimination is still open. However, special attention has to be payed to cut-simulation [86, 95], which may render cut-elimination as a pointless criterion.

Key insights:. Cut-elimination of a given object logic can often be reduced to showing the faithfulness of a shallow semantical embedding of this logic in HOL (with Henkin semantics); the approach has been applied to prove cut-elimination for some variants of quantified conditional logics, for which the question was still open; it should be possible to obtain similar cut-elimination results for many other challenging object logics by adopting the same reduction principle.

\subsection{Educational Study V: Lecture Course on Computational Metaphysics}

The early successes in the CompMeta project inspired the design of a worldwide new lecture course on computational metaphysics [96, 97. This lecture course, which was set-up and held in collaboration with Alexander Steen and Max Wisniewski, was awarded with the 2015/16 central teaching award of Freie Universität Berlin ${ }^{6}$ The course received substantial support from Jasmin Blanchette (Amsterdam), Wolfgang Lenzen (Osnabrück), Bruno WoltzenlogelPaleo (Canberra) and Edward Zalta (Stanford), who all contributed invited guest lectures.

Students with heterogenous knowledge backgrounds from computer science, mathematics, philosophy and physics attended the lecture course, and they came from all three major universities of Berlin: Freie Universität Berlin, Technical

\footnotetext{
${ }^{6}$ cf. http://www.fu-berlin.de/campusleben/lernen-und-lehren/2016/160428-lehrpreis/
} 
University Berlin and Humboldt University Berlin. The attendance in the lectures usually varied between 40 and 70 students. 36 students were formally registered for the course and were graded.

The steep learning curves of nearly all students were astonishing, in particular in the second half of the course, when small, heterogeneous student groups were formed to work each on an encoding and formal assessment of a different publication in philosophy or mathematics by adapting and utilising the SSE approach within the Isabelle/HOL proof assistant. The heterogeneous group compositions, the $24 / 7$ feedback from the Isabelle/HOL environment, and the motivating project topics were prime reasons, as the author believes, for the very good overall results of the course. A selection of project results has meanwhile been presented at conferences or published as book chapters or journal articles [98, 66, 74, 73, 99, 100, 67, 101. Several students picked up follow-up topics and turned them into BSc or MSc thesis projects [102, 103, 104, 105, 106.

A key ingredient for the successful implementation of the course has been, that a single methodology and overall technique (the SSE approach) was used throughout, enabling the students to quickly adopt a wide range of different logic variants in short time within a single proof assistant framework (Isabelle/HOL). The interdisciplinary course concept appears well suited to foster a much improved logic education across disciplines.

Summary of key insights. The SSE approach is well suited to support a novel form of university level logic education to heterogeneous groups of students; excellent learning curves are possible; new teaching methods are enabled in interaction with ITPs and ATPs for HOL.

\subsection{Further Results and Comments}

A range of related application studies (contributed partly also by collaborators) has not been mentioned above. Amongst others, these works include semantical embeddings of multivalued logic SIXTEEN [107], nominal logics [108, temporal logics [109, paraconsistent logics, intuitionistic modal logics, etc. Also the (partly ongoing) work of Streit, another student recruited from the computational metaphysics lecture course, on the formalisation of Boolos' textbook on provability logic [110] and on the formalisation of Bostrom's simulation argument [11] has not been addressed above.

A relevant and challenging future application direction of the semantical embedding approach lies in the modeling of legal, ethical, social and cultural norms in intelligent machines [112. To enable such applications, the author is currently, in a collaboration with Leon van der Torre and Xavier Parent from the University of Luxemburg, adapting the semantical embedding approach to cover recent developments in the area of deontic logics [113. Standard deontic logic, which is just a normal modal logic, is obviously already covered by the approach. More challenging has been the semantical embedding of e.g. input/output logic [114] and dyadic deontic logic [115]. First results in this application direction are promising [116, 117, 118, 119]. 


\subsubsection{Note on Invention and Creativity in ATPs}

As reported above, the theorem prover Leo-II detected the inconsistency of the axioms in Gödel's original variant of the ontological argument; this inconsistency was not known to philosophers before. The clue in the proof of falsity from the axioms [51, 50, is the empty essence lemma: from Gödel's [53. definition of essential properties (essence, cf. Ess. in Fig. 4) it follows that the empty property, i.e., the everywhere false property (alternatively we may pick the property of being self-different), is an essential property of every individual. Dana Scott [54] slightly modified Gödel's definition of essence in his variant of the ontological argument (for cosmetic reasons - the inconsistency was not known to him at the time), with the effect that the empty essence lemma is no longer valid.

In its successful, automatic discovery of the inconsistency, the Leo-II prover had to guess the instantiation of the empty property for a second-order variable during proof search [51, 50. This part in the proof is non-analytic: inspection shows that one cannot synthesise the required instantiation by e.g. unification with existing information (terms) in the search space. In fact, blind guessing of this instantiation seems unavoidable here. The author considers this as a small but nevertheless very interesting example for a true discovery (based on guessing and checking) by an ATP; and this discovery is philosophically relevant.

\subsubsection{Improved Infrastructure for HO Interactive and Automated Reasoning}

In the course of the above works, and in close collaboration with development of Leo-III, the CompMeta project fostered the development of a reusable theorem proving infrastructure for a range of non-classical logics 120, 121, 122, 123, 18. This includes various, reusable encodings in Isabelle/HOL syntax, Coq syntax and in TPTP syntax, which have been all made publicly available 7 Moreover, this includes a flexible pre-processing module [18, 124, 125. for the Leo-III prover (and any other TPTP THF 34] compliant prover). This preprocessor turns Leo-III into a flexible reasoner for a very wide range of propositional and quantified modal logics. In fact, no other implemented system is available today which covers a wider range of modal logic variants than Leo-III, and this approach can easily be extended for many other non-classical logics that have been mentioned above.

\section{Conclusion}

The presented reasoning framework based on shallow semantical embeddings in HOL constitutes the most widely applied universal logical reasoning approach available to date. There is, however, a significant difference to Leibniz' original idea of a characteristica universalis and to various related proposals in the literature. Instead of proposing a single, universal object-level formalism, the

\footnotetext{
${ }^{7}$ Cf. e.g. https://github.com/FormalTheology/GoedelGod
} 
shallow semantical embedding approach supports many different competing object logics from the logic zoo. No ontological commitment is enforced at the object logic level. For example, the approach well supports both classical and intuitionistic object logics, and can even elegantly combine them. The concrete portfolio of embedded object logics is determined by the specific requirements of an application at hand. Only at meta-level a single, unifying logic is provided, namely HOL (or any richer logic incorporating HOL). By unfolding the definitions of the logic embeddings, problem encodings utilising these object logics are uniformly mapped to meta-logic HOL. This way Leibniz' vision of a characteristica universalis is realised in an indirect way in the presented approach: universal logical reasoning is established (only) at the meta-level in HOL.

The presented universal logical reasoning framework has many challenging applications in artificial intelligence, computer science, philosophy, mathematics and natural language processing. A most relevant and timely application direction concerns the application of the semantical embedding approach for the modeling of ethical, legal, social and cultural norms in intelligent machines [116, ideally in combination with the realisation of human-intuitive forms of rational argumentation in machines complementing internal decision making means at the level of statistical information and subsymbolic representations.

Acknowledgements. A big thanks (in alphabetical order) goes to all collaborators and supporters of the CompMeta project, including (but not limited to): Jasmin Blanchette, Harold Boley, Frode Bjørdal, Chad Brown, Maximilian Claus, Ali Farjami, David Fuenmayor, Tobias Gleissner, Max Haslbeck, Daniel Kirchner, Hanna Lachnitt, Wolfgang Lenzen, Tomer Libal, Irina Makarenko, Paul Oppenheimer, Jens Otten, Xavier Parent, Larry Paulson, Florian Rabe, Raul Rojas, Fabian Schütz, Hans-Jörg Schurr, Dana Scott, Alexander Steen, David Streit, Geoff Sutcliffe, Leon van der Torre, Max Wisniewski, Bruno Woltzenlogel-Paleo, Edward Zalta, Marco Ziener.

\section{References}

[1] J. Lambek, P. Scott, Introduction to Higher Order Categorical Logic, Cambridge University Press, 1986.

[2] B. Jacobs, Categorical Logic and Type Theory, Vol. 141 of Studies in Logic and the Foundations of Mathematics, North Holland, Elsevier, 1999.

[3] H. Andreka, I. Németi, I. Sain, Universal Algebraic Logic, Studies in Universal Logic, Birkhäuser Basel, 2017.

[4] L. Moss, Coalgebraic logic, Annals of Pure and Applied Logic 96 (1-3) (1999) $277-317$.

[5] J. Rutten, Universal coalgebra: a theory of systems, Theoretical Computer Science 249 (1) (2000) 3-80. 
[6] W. Guttmann, G. Struth, T. Weber, Automating algebraic methods in Isabelle, in: S. Qin, Z. Qiu (Eds.), Proc. of ICFEM 2011, Vol. 6991 of LNCS, Springer, 2011, pp. 617-632.

[7] S. Foster, G. Struth, On the fine-structure of regular algebra, Journal of Automated Reasoning 54 (2) (2015) 165-197.

[8] A. Church, A formulation of the simple theory of types, Journal of Symbolic Logic 5 (1940) 56-68.

[9] P. Andrews, Church's type theory, in: E. N. Zalta (Ed.), The Stanford Encyclopedia of Philosophy, summer 2018 Edition, Metaphysics Research Lab, Stanford University, 2018.

[10] C. Benzmüller, D. Miller, Automation of higher-order logic, in: D. M. Gabbay, J. H. Siekmann, J. Woods (Eds.), Handbook of the History of Logic, Volume 9 - Computational Logic, North Holland, Elsevier, 2014, pp. 215-254. doi: 10.1016/B978-0-444-51624-4.50005-8.

[11] C. Benzmüller, L. Paulson, Quantified multimodal logics in simple type theory, Logica Universalis 7 (1) (2013) 7-20. doi:10.1007/s11787-012-0052-y

[12] G. Frege, Begriffsschrift. Eine der arithmetischen nachgebildete Formelsprache des reinen Denkens, Halle, 1879.

[13] P. B. Andrews, An Introduction to Mathematical Logic and Type Theory: To Truth Through Proof, Vol. 27 of Applied Logic Series, Springer, 2002.

[14] C. Benzmüller, C. Brown, M. Kohlhase, Higher-order semantics and extensionality, Journal of Symbolic Logic 69 (4) (2004) 1027-1088. doi:10.2178/jsl/ 1102022211 .

[15] T. Nipkow, L. C. Paulson, M. Wenzel, Isabelle/HOL: A Proof Assistant for Higher-Order Logic, no. 2283 in LNCS, Springer, 2002.

[16] Y. Bertot, P. Casteran, Interactive Theorem Proving and Program Development, Springer, 2004.

[17] C. Benzmüller, N. Sultana, L. C. Paulson, F. Theiß, The higher-order prover LEO-II, Journal of Automated Reasoning 55 (4) (2015) 389-404. doi:10.1007/ s10817-015-9348-y.

[18] A. Steen, C. Benzmüller, The higher-order prover Leo-III, in: D. Galmiche, S. Schulz, R. Sebastiani (Eds.), Automated Reasoning. IJCAR 2018, Vol. 10900 of LNCS, Springer, Cham, 2018, pp. 108-116. doi:10.1007/ 978-3-319-94205-6_8

[19] C. E. Brown, Satallax: An automatic higher-order prover, in: B. Gramlich, D. Miller, U. Sattler (Eds.), Automated Reasoning - 6th International Joint Conference, IJCAR 2012, Manchester, UK, June 26-29, 2012. Proceedings, Vol. 7364 of Lecture Notes in Computer Science, Springer, 2012, pp. 111-117. 
[20] J. C. Blanchette, T. Nipkow, Nitpick: A counterexample generator for higherorder logic based on a relational model finder, in: M. Kaufmann, L. C. Paulson (Eds.), Interactive Theorem Proving, First International Conference, ITP 2010, Edinburgh, UK, July 11-14, 2010. Proceedings, Vol. 6172 of Lecture Notes in Computer Science, Springer, 2010, pp. 131-146.

[21] J. C. Blanchette, S. Böhme, L. C. Paulson, Extending Sledgehammer with SMT solvers, Journal of Automated Reasoning 51 (1) (2013) 109-128.

[22] S. Schulz, System description: E 1.8, in: K. L. McMillan, A. Middeldorp, A. Voronkov (Eds.), Logic for Programming, Artificial Intelligence, and Reasoning - 19th International Conference, LPAR-19, Stellenbosch, South Africa, December 14-19, 2013. Proceedings, Vol. 8312 of Lecture Notes in Computer Science, Springer, 2013, pp. 735-743. doi:10.1007/978-3-642-45221-5.

[23] M. Deters, A. Reynolds, T. King, C. W. Barrett, C. Tinelli, A tour of CVC4: How it works, and how to use it, in: K. Claessen, V. Kuncak (Eds.), Formal Methods in Computer-Aided Design, FMCAD 2014, Lausanne, Switzerland, October 21-24, 2014, IEEE, 2014, p. 7.

[24] L. M. de Moura, N. Bjørner, Z3: An Efficient SMT Solver, in: C. R. Ramakrishnan, J. Rehof (Eds.), Tools and Algorithms for the Construction and Analysis of Systems, 14th International Conference, TACAS 2008, Held as Part of the Joint European Conferences on Theory and Practice of Software, ETAPS 2008, Budapest, Hungary, March 29-April 6, 2008. Proceedings, Vol. 4963 of Lecture Notes in Computer Science, Springer, 2008, pp. 337-340.

[25] J. C. Blanchette, A. Popescu, D. Wand, C. Weidenbach, More SPASS with Isabelle - Superposition with Hard Sorts and Configurable Simplification, in: L. Beringer, A. P. Felty (Eds.), Interactive Theorem Proving - Third International Conference, ITP 2012, Princeton, NJ, USA, August 13-15, 2012. Proceedings, Vol. 7406 of Lecture Notes in Computer Science, Springer, 2012, pp. $345-360$.

[26] L. Kovács, A. Voronkov, First-Order Theorem Proving and Vampire, in: N. Sharygina, H. Veith (Eds.), Computer Aided Verification - 25th International Conference, CAV 2013, Saint Petersburg, Russia, July 13-19, 2013. Proceedings, Vol. 8044 of Lecture Notes in Computer Science, Springer, 2013, pp. 1-35.

[27] M. Baldoni, Normal multimodal logics: Automatic deduction and logic programming extension, Ph.D. thesis, Dipartimento di Informatica, Universitá degli Studi di Torino, (Revised version dated July 9, 2003) (2003).

[28] R. Fagin, J. Y. Halpern, Y. Moses, M. Vardi, Reasoning About Knowledge, The MIT Press, 2004.

[29] H. Ohlbach, A. Nonnengart, M. de Rijke, D. Gabbay, Encoding two-valued nonclassical logics in classical logic, in: J. Robinson, A. Voronkov (Eds.), Handbook of Automated Reasoning (in 2 volumes), Elsevier and MIT Press, 2001, pp. 1403-1486.

[30] C. Benzmüller, Combining and automating classical and non-classical logics in classical higher-order logic, Annals of Mathematics and Artificial Intelligence 62 (1-2) (2011) 103-128. doi:10.1007/s10472-011-9249-7. 
[31] M. Sergot, Epistemic logic and 'common knowledge', Lecture Course Notes, Department of Computing Imperial College, London, https://www.doc.ic.ac. uk/ mjs/teaching/ModalTemporal499/Epistemic_499_v0809_2up.pdf (2008).

[32] A. Baltag, B. Renne, Dynamic epistemic logic, in: E. N. Zalta (Ed.), The Stanford Encyclopedia of Philosophy, winter 2016 Edition, Metaphysics Research Lab, Stanford University, 2016.

[33] C. Benzmüller, Comparing approaches to resolution based higher-order theorem proving, Synthese 133 (1-2) (2002) 203-235. doi:10.1023/A:1020840027781

[34] G. Sutcliffe, C. Benzmüller, Automated reasoning in higher-order logic using the TPTP THF infrastructure, Journal of Formalized Reasoning 3 (1) (2010) 1-27.

[35] J. Siekmann, C. Benzmüller, S. Autexier, Computer supported mathematics with OMEGA, Journal of Applied Logic 4 (4) (2006) 533-559. doi:10.1016/j. jal.2005.10.008

[36] S. Autexier, C. Benzmüller, D. Dietrich, J. Siekmann, OMEGA: Resourceadaptive processes in an automated reasoning systems, in: M. W. Crocker, J. Siekmann (Eds.), Resource-Adaptive Cognitive Processes, Cognitive Technologies, Springer, 2010, pp. 389-423. doi:10.1007/978-3-540-89408-7_17.

[37] J. Siekmann, C. Benzmüller, A. Fiedler, A. Meier, I. Normann, M. Pollet, Proof development in OMEGA: The irrationality of square root of 2, in: F. Kamareddine (Ed.), Thirty Five Years of Automating Mathematics, Applied Logic series (28), Kluwer Academic Publishers, 2003, pp. 271-314. doi:10.1007/978-94-017-0253-9_11

[38] C. Benzmüller, M. Schiller, J. Siekmann, Resource-bounded modelling and analysis of human-level interactive proofs, in: M. W. Crocker, J. Siekmann (Eds.), Resource-Adaptive Cognitive Processes, Cognitive Technologies, Springer, 2010, pp. 291-311. doi:10.1007/978-3-540-89408-7_13.

[39] C. Benzmüller, A. Pease, Higher-order aspects and context in SUMO, Journal of Web Semantics 12-13 (2012) 104-117. doi:10.1016/j.websem.2011.11.008

[40] C. Benzmüller, L. Paulson, Exploring properties of normal multimodal logics in simple type theory with LEO-II, in: C. Benzmüller, C. Brown, J. Siekmann, R. Statman (Eds.), Reasoning in Simple Type Theory - Festschrift in Honor of Peter B. Andrews on His 70th Birthday, Studies in Logic, Mathematical Logic and Foundations, College Publications, 2008, pp. 386-406, (Superseded by 2013 article in Logica Universalis).

[41] C. Benzmüller, L. Paulson, Multimodal and intuitionistic logics in simple type theory, The Logic Journal of the IGPL 18 (6) (2010) 881-892. doi:10.1093/ jigpal/jzp080

[42] E. N. Zalta, Principia Logico-Metaphysica (Draft/Excerpt), preprint available at https://mally.stanford.edu/principia.pdf (2018).

[43] R. C. Stalnaker, Mere Possibilities: Metaphysical Foundations of Modal Semantics, Princeton University Press, 2012. 
[44] T. Williamson, Modal Logic as Metaphysics, Oxford:OUP, 2013.

[45] B. Fitelson, E. N. Zalta, Steps toward a computational metaphysics, Journal Philosophical Logic 36 (2) (2007) 227-247. doi:10.1007/s10992-006-9038-7

[46] P. Oppenheimer, E. Zalta, A computationally-discovered simplification of the ontological argument, Australasian Journal of Philosophy 89 (2) (2011) 333349 .

[47] J. Alama, P. E. Oppenheimer, E. N. Zalta, Automating leibniz's theory of concepts, in: A. P. Felty, A. Middeldorp (Eds.), Automated Deduction - CADE-25 25th International Conference on Automated Deduction, Berlin, Germany, August 1-7, 2015, Proceedings, Vol. 9195 of Lecture Notes in Computer Science, Springer, 2015, pp. 73-97.

[48] J. Sobel, Logic and Theism: Arguments for and Against Beliefs in God, Cambridge U. Press, 2004.

[49] C. Benzmüller, B. Woltzenlogel Paleo, Automating Gödel's ontological proof of God's existence with higher-order automated theorem provers, in: T. Schaub, G. Friedrich, B. O'Sullivan (Eds.), ECAI 2014, Vol. 263 of Frontiers in Artificial Intelligence and Applications, IOS Press, 2014, pp. 93 - 98. doi:10.3233/ 978-1-61499-419-0-93.

[50] C. Benzmüller, B. Woltzenlogel Paleo, The inconsistency in Gödel's ontological argument: A success story for AI in metaphysics, in: S. Kambhampati (Ed.), IJCAI 2016, Vol. 1-3, AAAI Press, 2016, pp. 936-942.

URL http://www . ijcai .org/Proceedings/16/Papers/137.pdf

[51] C. Benzmüller, B. Woltzenlogel Paleo, An object-logic explanation for the inconsistency in Gödel's ontological theory (extended abstract, sister conferences), in: M. Helmert, F. Wotawa (Eds.), KI 2016: Advances in Artificial Intelligence, Proceedings, LNCS, Springer, Berlin, Germany, 2016, pp. 244-250. doi: $10.1007 / 978-3-319-46073-4$

[52] C. Benzmüller, B. Woltzenlogel Paleo, Experiments in Computational Metaphysics: Gödel's proof of God's existence, Savijnanam: scientific exploration for a spiritual paradigm. Journal of the Bhaktivedanta Institute 9 (2017) 43-57.

[53] K. Gödel, Appx. A: Notes in Kurt Gödel's Hand, in: J. Sobel (Ed.), Logic and Theism: Arguments for and Against Beliefs in God, Cambridge U. Press, 1970, pp. $144-145$.

[54] D. Scott, Appx. B: Notes in Dana Scott's Hand, in: J. Sobel (Ed.), Logic and Theism: Arguments for and Against Beliefs in God, Cambridge U. Press, 1972, pp. $145-146$.

[55] Y. Bertot, P. Casteran, Interactive Theorem Proving and Program Development - Coq'Art: The Calculus of Inductive Constructions, Texts in Theoretical Computer Science, Springer, 2004.

[56] J. Sobel, Gödel's ontological proof, in: On Being and Saying. Essays for Richard Cartwright, MIT Press, 1987, pp. 241-261. 
[57] C. Anderson, Some emendations of Gödel's ontological proof, Faith and Philosophy $7(3)$.

[58] A. Anderson, M. Gettings, Gödel ontological proof revisited, in: Gödel'96: Logical Foundations of Mathematics, Computer Science, and Physics: Lecture Notes in Logic 6, Springer, 1996, pp. 167-172.

[59] P. Hájek, Magari and others on Gödel's ontological proof, in: A. Ursini, P. Agliano (Eds.), Logic and algebra, Dekker, New York etc., 1996, p. 125-135.

[60] P. Hájek, Der Mathematiker und die Frage der Existenz Gottes, in: B. Buldt et al. (Ed.), Kurt Gödel. Wahrheit und Beweisbarkeit, öbv \& hpt, Wien, 2001, pp. 325-336, iSBN 3-209-03835-X.

[61] P. Hájek, A new small emendation of Gödel's ontological proof, Studia Logica 71 (2) (2002) 149-164.

[62] F. Bjørdal, Understanding Gödel's ontological argument, in: T. Childers (Ed.), The Logica Yearbook 1998, Filosofia, 1999, pp. 214-217.

[63] C. Benzmüller, L. Weber, B. Woltzenlogel Paleo, Computer-assisted analysis of the Anderson-Hájek controversy, Logica Universalis 11 (1) (2017) 139-151. doi:10.1007/s11787-017-0160-9.

[64] C. Benzmüller, B. Woltzenlogel Paleo, Higher-order modal logics: Automation and applications, in: A. Paschke, W. Faber (Eds.), Reasoning Web 2015, no. 9203 in LNCS, Springer, Berlin, Germany, 2015, pp. 32-74. doi:10.1007/ 978-3-319-21768-0_2

[65] C. Benzmüller, B. Woltzenlogel Paleo, The modal collapse as a collapse of the modal square of opposition, in: J.-Y. Béziau, G. Basti (Eds.), The Square of Opposition: A Cornerstone of Thought (Collection of papers related to the World Congress on the Square of Opposition IV, Vatican, 2014), http://www.springer.com/us/book/9783319450612, Studies in Universal Logic, Springer International Publishing Switzerland, 2016, pp. 307-313. doi:10.1007/978-3-319-45062-9_18

[66] D. Fuenmayor, C. Benzmüller, Types, Tableaus and Gödel's God in Isabelle/HOL Archive of Formal Proofs 2017.

URL http://afp.sourceforge.net/entries/Types_Tableaus_and_Goedels_ God.shtml

[67] A. Steen, M. Wisniewski, C. Benzmüller, Agent-based HOL reasoning, in: G.-M. Greuel, T. Koch, P. Paule, A. Sommese (Eds.), Mathematical Software - ICMS 2016, 5th International Congress, Proceedings, Vol. 9725 of LNCS, Springer, Berlin, Germany, 2016, pp. 75-81. doi:10.1007/978-3-319-42432-3_10.

[68] M. Fitting, Types, Tableaus, and Gödel's God, Kluwer, 2002.

[69] A. N. Whitehead, B. Russell, Principia Mathematica, 3 vols, Cambridge: Cambridge University Press. Second edition, 1925 (Vol. 1), 1927 (Vols 2, 3). Abridged as Principia Mathematica to *56, Cambridge: Cambridge University Press, 1962., 1910, 1912, 1913. 
[70] E. Zalta, Intensional Logic and the Metaphysics of Intentionality, A Bradford book, MIT Press, 1988.

[71] E. Zalta, Abstract Objects: An Introduction to Axiomatic Metaphysics, Synthese Library, Springer, 1983.

[72] P. E. Oppenheimer, E. N. Zalta, Relations versus functions at the foundations of logic: Type-theoretic considerations, Journal of Logic and Computation 21 (2) (2011) 351-374. doi:10.1093/logcom/exq017. URL http://dx.doi.org/10.1093/logcom/exq017

[73] D. Kirchner, Representation and partial automation of the principia logicometaphysica in Isabelle/HOL, Archive of Formal Proofs 2017.

URL https://www.isa-afp.org/entries/PLM.html

[74] D. Kirchner, C. Benzmüller, E. N. Zalta, Mechanizing principia logicometaphysica in functional type theory, Tech. rep., CoRR, preprint of submitted article (2017).

URL https://arxiv.org/abs/1711.06542

[75] G. Link (Ed.), One Hundred Years of Russell's Paradox, De Gruyter, 2008.

[76] G. Frege, Begriffsschrift, eine der arithmetischen nachgebildete Formelsprache des reinen Denkens, Halle, 1879, translated in [? ].

[77] K. Lambert, The definition of e(xistence)! in free logic, in: Abstracts: The International Congress for Logic, Methodology and Philosophy of Science, Stanford: Stanford University Press, 1960.

[78] D. Scott, Existence and description in formal logic, in: R. Schoenman (Ed.), Bertrand Russell: Philosopher of the Century, George Allen \& Unwin, London, 1967, pp. 181-200, (Reprinted with additions in: Philosophical Application of Free Logic, edited by K. Lambert. Oxford Universitry Press, 1991, pp. 28 - 48).

[79] K. Lambert, Free Logic: Selected Essays, Cambridge: Cambridge University Press, 2002.

[80] J. Nolt, Free logic, in: E. N. Zalta (Ed.), The Stanford Encyclopedia of Philosophy, fall 2018 Edition, Metaphysics Research Lab, Stanford University, 2018.

[81] C. Benzmüller, D. S. Scott, Axiomatizing category theory in free logic, Tech. rep., CoRR (2016).

URL http://arxiv.org/abs/1609.01493

[82] C. Benzmüller, D. S. Scott, Automating free logic in Isabelle/HOL, in: G.-M. Greuel, T. Koch, P. Paule, A. Sommese (Eds.), Mathematical Software - ICMS 2016, 5th International Congress, Proceedings, Vol. 9725 of LNCS, Springer, Berlin, Germany, 2016, pp. 43-50. doi:10.1007/978-3-319-42432-3_6

[83] C. Benzmüller, D. S. Scott, Axiom systems for category theory in free logic Archive of Formal Proofs 2018. URL https://www .isa-afp.org/entries/AxiomaticCategoryTheory.html

[84] P. Freyd, A. Scedrov, Categories, Allegories, North Holland, 1990. 
[85] D. Scott, Identity and existence in intuitionistic logic, in: M. Fourman, C. Mulvey, D. Scott (Eds.), Applications of Sheaves: Proceedings of the Research Symposium on Applications of Sheaf Theory to Logic, Algebra, and Analysis, Durham, July 9-21, 1977, Vol. 752 of Lecture Notes in Mathematics, Springer Berlin Heidelberg, 1979, pp. 660-696.

[86] C. Benzmüller, Cut-elimination for quantified conditional logic, Journal of Philosophical Logic 46 (3) (2017) 333-353. doi:10.1007/s10992-016-9403-0.

[87] R. C. Stalnaker, A theory of conditionals, in: Studies in Logical Theory, Blackwell, 1968, pp. 98-112.

[88] B. Chellas, Basic conditional logic, Journal of Philosophical Logic 4 (2) (1975) $133-153$.

[89] J. Delgrande, On first-order conditional logics, Artificial Intelligence 105 (1-2) (1998) 105-137.

[90] J. Delgrande, A first-order conditional logic for prototypical properties, Artificial Intelligence 33 (1) (1987) 105-130.

[91] N. Friedman, J. Halpern, D. Koller, First-order conditional logic for default reasoning revisited, ACM Transactions on Computational Logic 1 (2) (2000) $175-207$.

[92] D. Pattinson, L. Schröder, Generic modal cut elimination applied to conditional logics, Logical Methods in Computer Science 7 (1). doi:10.2168/LMCS-7(1: 4) 2011 .

[93] J. Rasga, Sufficient conditions for cut elimination with complexity analysis, Ann. Pure Appl. Logic 149 (1-3) (2007) 81-99. doi:10.1016/j.apal.2007.08.001.

[94] C. Benzmüller, Automating quantified conditional logics in HOL, in: F. Rossi (Ed.), 23rd International Joint Conference on Artificial Intelligence (IJCAI-13), AAAI Press, Beijing, China, 2013, pp. 746-753.

[95] C. Benzmüller, C. Brown, M. Kohlhase, Cut-simulation and impredicativity, Logical Methods in Computer Science 5 (1:6) (2009) 1-21. doi:10.2168/ LMCS-5(1:6) 2009

[96] M. Wisniewski, A. Steen, C. Benzmüller, Einsatz von Theorembeweisern in der Lehre, in: A. Schwill, U. Lucke (Eds.), Hochschuldidaktik der Informatik: 7. Fachtagung des GI-Fachbereichs Informatik und Ausbildung/Didaktik der Informatik; 13.-14. September 2016 an der Universität Potsdam, Commentarii informaticae didacticae (CID), Universitätsverlag Potsdam, Potsdam, Germany, 2016, pp. 81-92.

[97] C. Benzmüller, M. Wisniewski, A. Steen, Computational Metaphysics, this lecture course proposal received the 2015 central teaching award of FU Berlin (2015).

URL http://christoph-benzmueller.de/papers/R57.pdf 
[98] D. Fuenmayor, C. Benzmüller, A case study on computational hermeneutics: E.J. Lowe's Modal ontological argument, Journal of Applied Logic - IfCoLoG Journal of Logics and their Applications (special issue on Formal Approaches to the Ontological Argument)Accepted for publication; to be published also as chapter in the book 'Beyond Faith and Rationality: Essays on Logic, Religion and Philosophy' printed in the Springer book series 'Sophia Studies in Crosscultural Philosophy of Traditions and Cultures'.

URL http://christoph-benzmueller.de/papers/J38.pdf

[99] M. Bentert, C. Benzmüller, D. Streit, B. Woltzenlogel Paleo, Analysis of an ontological proof proposed by Leibniz, in: C. Tandy (Ed.), Death and Anti-Death, Volume 14: Four Decades after Michael Polanyi, Three Centuries after G.W. Leibniz, Ria University Press, 2016, preprint: http://christoph-benzmueller. de/papers/B16.pdf

[100] D. Fuenmayor, C. Benzmüller, Automating emendations of the ontological argument in intensional higher-order modal logic, in: KI 2017: Advances in Artificial Intelligence 40th Annual German Conference on AI, Dortmund, Germany, September 25-29, 2017, Proceedings, Vol. 10505 of LNAI, Springer, 2017, pp. 114-127. doi:10.1007/978-3-319-67190-1_9.

[101] D. Fuenmayor, C. Benzmüller, Computer-assisted Reconstruction and Assessment of E. J. Lowe's Modal Ontological Argument, Archive of Formal Proofs 2017.

URL https://www.isa-afp.org/entries/Lowe_Ontological_Argument.html

[102] I. Makarenko, Automatisierung von freier logik in logik höherer stufe, Bachelor's thesis, Department of Mathematics and Computer Science, Freie Universität Berlin (2016).

[103] D. Fuenmayor, Computational hermeneutics: Using computers to understand philosophical arguments, Bachelor's thesis, Department of Philosophy, Freie Universität Berlin (2017).

[104] T. Gleissner, Converting higher-order modal logic problems into classical higherorder logic, Bachelor's thesis, Department of Mathematics and Computer Science, Freie Universität Berlin (2017).

[105] D. Kirchner, Mechanization of the principia-logico metaphysica, Master's thesis, Department of Mathematics and Computer Science, Freie Universität Berlin (2017).

[106] F. Schütz, Wahrhaftiger Toren Zorn - Repräsentation und Interpretation von Argumenten, Master's thesis, Department of Mathematics and Computer Science, Freie Universität Berlin (2017).

[107] A. Steen, C. Benzmüller, Sweet SIXTEEN: Automation via embedding into classical higher-order logic, Logic and Logical Philosophy 25 (2016) 535-554. doi:10.12775/LLP.2016.021.

[108] M. Wisniewski, A. Steen, Embedding of quantified higher-order nominal modal logic into classical higher-order logic, in: C. Benzmüller, J. Otten (Eds.), 1st International Workshop on Automated Reasoning in Quantified Non-Classical 
Logics (ARQNL 2014), Vienna, Austria, Proceedings, Vol. 33 of EasyChair Proceedings in Computing, EasyChair Publications, 2014, pp. 59-64.

[109] M. Claus, Software model checking with higher-order automated theorem provers: A logic embedding approach, Master's thesis, Department of Mathematics and Computer Science, Freie Universität Berlin (2015).

[110] G. Boolos, The Logic of Provability, Cambridge University Press, 1993.

[111] N. Bostrom, Are we living in a computer simulation?, The Philosophical Quarterly 53 (211) (2003) 243-255. doi:10.1111/1467-9213.00309.

[112] C. Benzmüller, Universal reasoning, rational argumentation and human-machine interaction, Tech. rep., CoRR (2017). URL http://arxiv.org/abs/1703.09620

[113] D. Gabbay, J. Horty, X. Parent, R. van der Meyden, L. van der Torre (Eds.), Handbook of Deontic Logic and Normative Systems, College Publications, 2013.

[114] D. Makinson, L. W. N. van der Torre, Input/output logics, Journal of Philosophical Logic 29 (4) (2000) 383-408. doi:10.1023/A:1004748624537.

[115] J. Carmo, A. J. I. Jones, Completeness and decidability results for a logic of contrary-to-duty conditionals, Journal of Logic and Computation 23 (3) (2013) 585-626. doi:10.1093/logcom/exs009.

[116] C. Benzmüller, X. Parent, L. van der Torre, A deontic logic reasoning infrastructure, in: F. Manea, R. G. Miller, D. Nowotka (Eds.), 14th Conference on Computability in Europe, CiE 2018, Kiel, Germany, July 30-August, 2018, Proceedings, Vol. 10936 of LNCS, Springer, 2018, pp. 60-69. doi: 10.1007/978-3-319-94418-0_6

[117] C. Benzmüller, X. Parent, First experiments with a flexible infrastructure for normative reasoning, Tech. rep., CoRR (2018).

URL http://arxiv.org/abs/1804.02929

[118] C. Benzmüller, X. Parent, I/O logic in HOL - first steps, Tech. rep., CoRR (2018).

URL http://arxiv .org/abs/1803.09681

[119] C. Benzmüller, A. Farjami, X. Parent, Faithful semantical embedding of a dyadic deontic logic in HOL, Tech. rep., CoRR (2018).

URL https://arxiv .org/abs/1802.08454

[120] C. Benzmüller, J. Otten, T. Raths, Implementing and evaluating provers for first-order modal logics, in: L. D. Raedt, C. Bessiere, D. Dubois, P. Doherty, P. Frasconi, F. Heintz, P. Lucas (Eds.), ECAI 2012, Vol. 242 of Frontiers in Artificial Intelligence and Applications, IOS Press, Montpellier, France, 2012, pp. 163-168. doi:10.3233/978-1-61499-098-7-163.

[121] C. Benzmüller, T. Raths, HOL based first-order modal logic provers, in: K. L. McMillan, A. Middeldorp, A. Voronkov (Eds.), Proceedings of the 19th International Conference on Logic for Programming, Artificial Intelligence and Reasoning (LPAR), Vol. 8312 of LNCS, Springer, Stellenbosch, South Africa, 2013, pp. 127-136. doi:10.1007/978-3-642-45221-5_9 
[122] M. Wisniewski, A. Steen, C. Benzmüller, TPTP and beyond: Representation of quantified non-classical logics, in: C. Benzmüller, J. Otten (Eds.), ARQNL 2016. Automated Reasoning in Quantified Non-Classical Logics, Vol. 1770, CEUR Workshop Proceedings, http://ceur-ws.org, 2016, pp. 51-65.

[123] T. Gleißner, A. Steen, C. Benzmüller, Theorem provers for every normal modal logic, in: T. Eiter, D. Sands (Eds.), LPAR-21. 21st International Conference on Logic for Programming, Artificial Intelligence and Reasoning, Vol. 46 of EPiC Series in Computing, EasyChair, Maun, Botswana, 2017, pp. 14-30. doi:10. $29007 / j$ jb9.

[124] C. Benzmüller, A. Steen, M. Wisniewski, Leo-III version 1.1 (system description), in: T. Eiter, D. Sands, G. Sutcliffe, A. Voronkov (Eds.), IWIL@LPAR 2017 Workshop and LPAR-21 Short Presentations, Maun, Botswana, May 7-12, 2017, Vol. 1 of Kalpa Publications in Computing, EasyChair, Maun, Botswana, 2017, pp. 11-61.

[125] A. Steen, M. Wisniewski, C. Benzmüller, Going polymorphic - TH1 reasoning for Leo-III, in: T. Eiter, D. Sands, G. Sutcliffe, A. Voronkov (Eds.), IWIL@LPAR 2017 Workshop and LPAR-21 Short Presentations, Maun, Botswana, May 7-12, 2017, Vol. 1 of Kalpa Publications in Computing, EasyChair, Maun, Botswana, 2017, pp. 100-112. 